\title{
Comment on "Collective modes and gapped momentum states in liquid Ga: Experiment, theory, and simulation"
}

\author{
Taras Bryk ${ }^{1,2}$, Ihor Mryglod ${ }^{1}$, Giancarlo Ruocco ${ }^{3,4}$ \\ 1 Institute for Condensed Matter Physics, \\ National Academy of Sciences of Ukraine, \\ UA-79011 Lviv, Ukraine \\ ${ }^{2}$ Institute of Applied Mathematics and Fundamental Sciences, \\ Lviv National Polytechnic University, UA-79013 Lviv, Ukraine \\ ${ }^{3}$ Center for Life Nano Science @Sapienza, Istituto Italiano di Tecnologia, \\ 295 Viale Regina Elena, I-00161, Roma, Italy and \\ ${ }^{4}$ Dipartimento di Fisica, Universita' di Roma "La Sapienza", I-00185, Roma, Italy
}

(Dated: August 13, 2020)

\begin{abstract}
We show that the presented in [1] theoretical expressions for longitudinal current spectral function $C^{L}(k, \omega)$ and dispersion of collective excitations are not correct. Indeed, they are not compatible with the continuum limit and $C^{L}(k, \omega \rightarrow 0)$ contradicts the continuity equation.
\end{abstract}




\section{INTRODUCTION}

In a recent paper [1] the authors formulated their "overarching goal of this research programme ... to reach the stage where, despite the complexity of their theoretical description, liquids emerge as systems amenable to theoretical understanding at the level comparable to gases and solids". Looking at Figs.4 and 5 of [1] one can really make sure that the authors of [1] reached their ambitious goal in perfect agreement between the proposed theory and computer simulations. In this paper the authors proposed theoretical expressions for the longitudinal current spectral function $C^{L}(k, \omega)$, with $k$ and $\omega$ being wave number and frequency, and for the dispersion of longitudinal collective excitations $\omega_{c}^{L}(k)$. Their expressions for $C^{L}(k, \omega)$ (Eq.18) and $\omega_{c}^{L}(k)$ (Eq.20), as one can judge from their Figs.4 and 5, recover with high precision the molecular dynamics (MD) data in a wide range of wave numbers and temperatures. The $C^{L}(k, \omega)$ in their theoretical scheme was obtained from a simple continued fraction shown in their Eq.11. Although the standard approach for description of collective dynamics in liquids is to represent the Laplace-transformed density-density time correlation function as a continued fraction[2, 3], in [1] the authors derived the continued fraction for the longitudinal currrent-current correlations. Applying different closures for the chain of memory functions like in [4-6] one can obtain formal solution for $C^{L}(k, \omega)$ within a precision of several its frequency moments.

However, such an approach of Ref.[1] is not really consistent with the hydrodynamics[4, 7], which is a collection of local conservation laws. Any liquid system on the spatial scales much larger than the mean interatomic distance must behave similarly from the point of view of slow collective modes derived by fluctuations of conserved quantities. In [1] the proposed theoretical approch is developed from a single conserved dynamic variable, longitudinal component of total momentum $J^{L}(k, t)$, which is the slowest dynamic variable in the presented approach. It is well known from the textbooks $[4,7]$ as well as from other multivariable approaches [8-10] which dynamic variables are responsible for description of the viscoelastic transition in dispersion of collective excitations [11, 12]. The theoretical approach [1] does not contain coupling of longitudinal current fluctuations with the fluctuations of other conserved quantities, namely density $n(k, t)$ and energy $e(k, t)$ ones. The energy (or heat) density fluctuations reflect specific for liquids fluctuations of local temperature[13], and long-wavelength heat relaxation processes are responsible for the central Rayleigh peak of 
the dynamic structure factor $S(k, \omega)$ for one-component liquids at sufficiently small wave numbers $k$. Outside the hydrodynamic regime the short-wavelength density fluctuations $n(k, t)$ reflect the processes connected with structural relaxation and instead of heat relaxation form the leading contribution to the central peak of $S(k, \omega)[12,14,15]$. The presence of heat and density relaxation, therefore, are essential ingredients for a correct description of the spectra, including the propagating density fluctuations regions, which are the main target of Ref.[1].

The poor theoretical approach presented in Ref.[1], missing the coupling with the most important for liquids slow processes, is an oversimplified theory. It is, therefore, difficult to understand why it is able to reproduce to a very good degree of accuracy the molecular dynamics (MD) data for $C^{L}(k, \omega)$ in some region of wave numbers as it is shown in their Fig.4 [1]. Moreover, we were motivated to understand why their expressions were able to recover the adiabatic speed of sound in the long-wavelength region of their Fig.5. Our question was: is it possible within the proposed fit-free theoretical scheme to obtain in the long-wavelength limit the propagating modes with adiabatic speed of sound $c_{s}$ ? The multivariable approaches

based on the set of dynamic variables $\left\{J^{L}(k, t), \dot{J}^{L}(k, t), \ldots\right\}$ usually can produce in the longwavelength limit the propagating modes only in elastic regime with propagation speed being the high-frequency one $c_{\infty}$ slightly renormalized due to the coupling to faster kinetic modes. No viscoelastic effects like positive sound dispersion can be expected in this theory.

Motivated by the surprisingly good agreement shown in their Fig.4 we will check the expressions (Eqs.17-20) of [1] and behavior of their "relaxation parameters" $\Delta_{i}(k)$ in the $k \rightarrow 0$ limit using a simple Lennard-Jones fluid, because of its simplicity in order to have analytical spacial derivatives of inteparticle potential needed for calculations of $\Delta_{i}(k)$ and their Eqs.17-20. In the next Section we provide details of our MD simulations and calculations of corresponding correlators. Then we will present our resuts and discuss them in comparison with the Eqs.17-20 of [1]. The last Section contains conclusion of this study.

\section{DETAILS OF MD SIMULATIONS}

We performed molecular dynamics simulations for supercritical $\mathrm{Ne}$ at $\mathrm{T}=295 \mathrm{~K}$ and density $1600 \mathrm{~kg} / \mathrm{m}^{3}$ using its Lennard-Jones potentials the same as in our previous study [16]. A model system of 4000 particles was simulated in microcanonical ensemble with perfect 

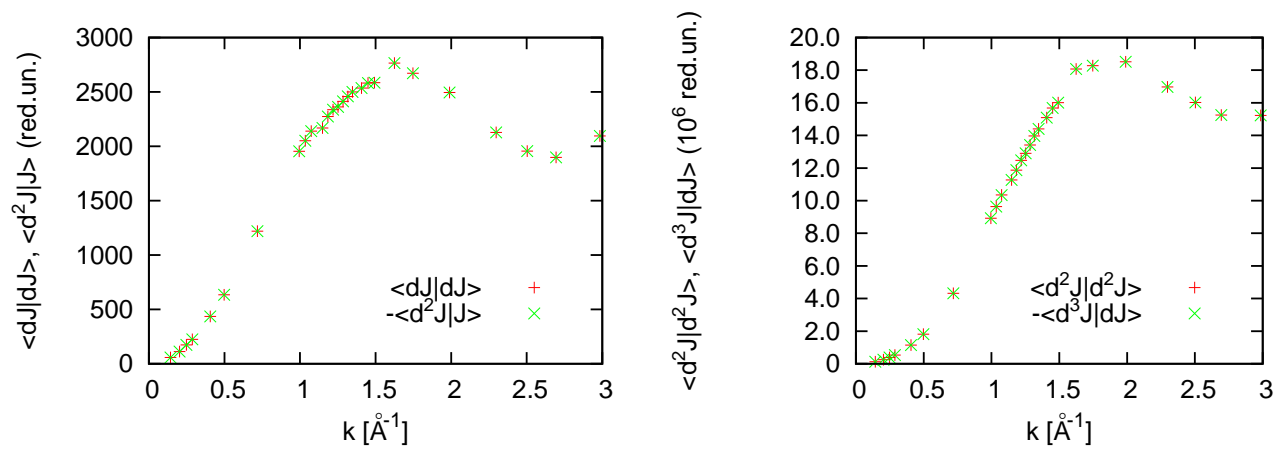

FIG. 1: Check of the properties of time derivatives of longitudinal current for static correlators $\left\langle\dot{J}_{L}(-k) \dot{J}_{L}(k)\right\rangle \equiv-\left\langle\ddot{J}_{L}(-k) J_{L}(k)\right\rangle(\mathrm{a})$ and $\left\langle\ddot{J}_{L}(-k) \ddot{J}_{L}(k)\right\rangle \equiv-\left\langle\dddot{J}_{L}(-k) \dot{J}_{L}(k)\right\rangle$ (b) for supercritical Ne at $\mathrm{T}=295 \mathrm{~K}$ and density $1600 \mathrm{~kg} / \mathrm{m}^{3}$.

energy conservation over the whole production run of 300000 time steps. The time step was 0.5 fs. Our main task was in sampling the space-Fourier components of all hydrodynamic variables, i.e. of density $n(k, t)$, mass-current $\mathbf{J}(k, t)$ and energy $e(k, t)$, as well as of their time derivatives, in particular, of the mass-current up to the third order $\dddot{\mathbf{J}}(k, t)$. We sampled all the possible wave vectors corresponding to the same absolute value, and used all them in spherical average of the corresponding correlators. The smallest wave number sampled in this MD study was $0.143598 \AA^{-1}$.

In order to check reliability of the sampled time derivatives of the longitudinal masscurrent and of our calculated static correlators we made use of the exact relations, which follow from a property of time derivatives of time correlations functions [4]

$$
\begin{gathered}
\left\langle\dot{J}_{L}(-k) \dot{J}_{L}(k)\right\rangle \equiv-\left\langle\ddot{J}_{L}(-k) J_{L}(k)\right\rangle \\
\left\langle\ddot{J}_{L}(-k) \ddot{J}_{L}(k)\right\rangle \equiv-\left\langle\dddot{J}_{L}(-k) \dot{J}_{L}(k)\right\rangle .
\end{gathered}
$$

One can see in Fig. 1 that perfect equivalence (difference less than $0.2 \%$ for any $k$-point) is the evidence of correct direct sampling of $J_{L}(k, t), \dot{J}_{L}(k, t) \ddot{J}_{L}(k, t)$ and $\dddot{J}_{L}(k, t)$ in $\mathrm{MD}$ simulations. These dynamic variables are needed for calculations of quantities $\Delta_{i}(k), i=$ 1,2,3 in expressions for $C^{L}(k, \omega)$ and $\omega_{c}^{L}(k)$ in [1]. Throughout this paper we will use reduced units of energy $k_{B} T=1$, mass $m=1$ and time $\tau_{\sigma}=1.997446 \mathrm{ps}$ 


\section{RESULTS AND DISCUSSION}

As we mentioned above the perfect agreement between the proposed in [1] fit-free theory and MD results for $C^{L}(k, \omega)$ in their Fig.4 looks too good to be true. Indeed, a simplest check of their Eq.18 in the $\omega \rightarrow 0$ limit results in the non-zero value of $C^{L}(k, \omega=0)$

$$
C^{L}(k, \omega=0)=\frac{1}{\pi} \frac{\Delta_{1}(k) \Delta_{2}(k) \Delta_{3}(k)^{3 / 2}}{B_{0}(k)} \equiv \frac{1}{\pi} \frac{\Delta_{2}(k)}{\Delta_{1}(k) \Delta_{3}(k)^{1 / 2}},
$$

while any viscoelastic theory must result in $C^{L}(k, \omega=0) \equiv 0$ as the consequence of continuity equation. We cannot explain how the authors [1] obtained in their Fig.4 the $C^{L}(k, \omega \rightarrow 0) \propto \omega^{2}$ behavior from their fit-free theory (their Eq.18).

We calculated from their Eqs.16-17 the "relaxation parameters" $\Delta_{i}(k), i=1,2,3$ and doublechecked the relations:

$$
\Delta_{1}(k)+\Delta_{2}(k)=\frac{\langle\ddot{J}(-k) \ddot{J}(k)\rangle}{\langle\dot{J}(-k) \dot{J}(k)\rangle},
$$

where the right hand side tends to a constant in long-wavelength limit and is simply the ratio of $k$-dependences shown in Fig.1(a,b), and

$$
\Delta_{3}(k)=\left[\langle\dddot{J}(-k) \dddot{J}(k)\rangle-\frac{\left\langle\ddot{J}_{L}(-k) \ddot{J}_{L}(k)\right\rangle^{2}}{\left\langle\dot{J}_{L}(-k) \dot{J}_{L}(k)\right\rangle}\right] /\left[\left\langle\ddot{J}_{L}(-k) \ddot{J}_{L}(k)\right\rangle-\frac{\left\langle\dot{J}_{L}(-k) \dot{J}_{L}(k)\right\rangle^{2}}{\left\langle J_{L}(-k) J_{L}(k)\right\rangle}\right] .
$$

In Fig. 2 we show the $k$-dependence of the "relaxation parameters"[1] and one can see the parameters $\Delta_{2}(k)$ and $\Delta_{3}(k)$ tending in the long-wavelength limit to non-zero values while

$$
\Delta_{1}(k) \equiv \frac{\left\langle\dot{J}_{L}(-k) \dot{J}_{L}(k)\right\rangle}{\left\langle J_{L}(-k) J_{L}(k)\right\rangle}
$$

behaves in $k \rightarrow 0$ limit as $\propto c_{\infty}^{2} k^{2}$ with $c_{\infty}$ being the high-frequency speed of sound.

Now we can estimate how large is the deviation of $C^{L}(k, \omega=0)$ from the correct zero value. Since the $\Delta_{1}(k)$ goes to zero in the long-wavelength limit and $\Delta_{2}(k \rightarrow 0)$ and $\Delta_{3}(k \rightarrow 0)$ tend to finite non-zero values, the resulting $C^{L}(k, \omega=0)$ taken from Eq.18 of [1] should diverge for $k \rightarrow 0$. Indeed, in Fig.3 one can observe the strong increase of $C^{L}(k, \omega=0) \propto k^{-2}$ in [1], that means wrong theoretical result comparing with the exact relation $C^{L}(k, \omega \rightarrow 0)=0$.

Now we will analyze the expression for dispersion of collective excitations [1]. Since only the "relaxation parameter" $\Delta_{1}(k)$ tends to zero as $k^{2}$ in the long-wavelength limit, 


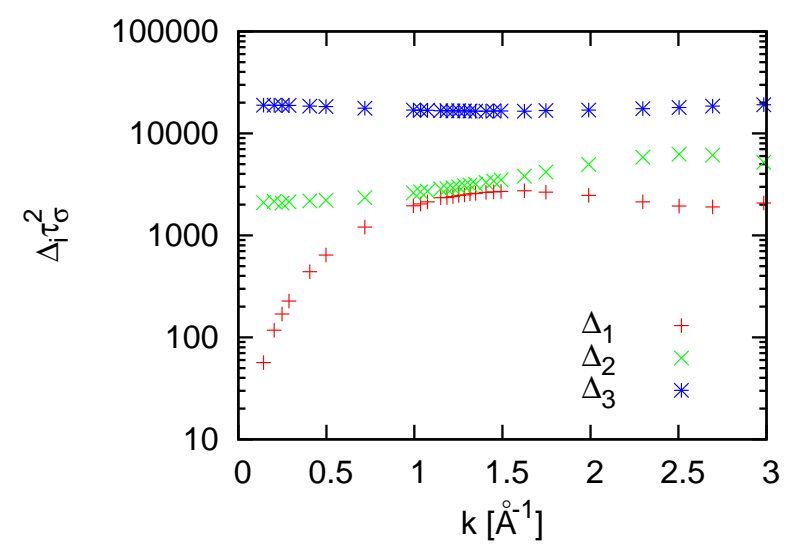

FIG. 2: Dependence of the "relaxation parameters" $\Delta_{i}, i=1,2,3$ (Eq.16 of [1]) on wave numbers for supercritical $\mathrm{Ne}$ at $\mathrm{T}=295 \mathrm{~K}$ and density $1600 \mathrm{~kg} / \mathrm{m}^{3}$.

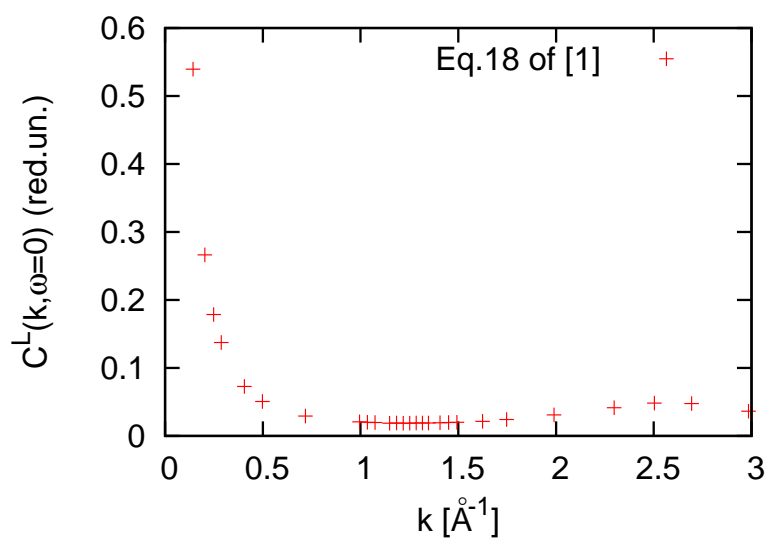

FIG. 3: Dependence of the zero-frequency value $C^{L}(k, \omega=0)$ (Eq.18 of [1]) on wave numbers for supercritical Ne at $\mathrm{T}=295 \mathrm{~K}$ and density $1600 \mathrm{~kg} / \mathrm{m}^{3}$.

and higher "relaxation parameters" $\Delta_{2,3}(k)$ tend to constants in that limit, one can easily estimate, that their Eq.(20) for $\omega_{c}^{L}(k)$ tends to a constant for $k \rightarrow 0$

$$
\omega_{c}^{L}(k \rightarrow 0)=\frac{\Delta_{2}(0)}{\sqrt{2\left[\Delta_{3}(0)-\Delta_{2}(0)\right]}},
$$

while the correct dispersion law had to recover in that limit the hydrodynamic dispersion law $\omega(k \rightarrow 0)=c_{s} k$. In Fig. 4 we show the dispersion of collective acoustic modes estimated from the peak positions of MD-derived $C^{L}(k, \omega)$ (plus symbols with error bars) and compare it with the dispersion of "bare" (non-damped) high-frequency modes which in the longwavelength limit have linear dispersion with the high-frequency (elastic) speed of sound 
$c_{\infty}$

$$
\omega_{\infty}(k \rightarrow 0)=\left.\left[\frac{\left\langle\dot{J}_{L}(-k) \dot{J}_{L}(k)\right\rangle}{\left\langle J_{L}(-k) J_{L}(k)\right\rangle}\right]^{1 / 2}\right|_{k \rightarrow 0} \rightarrow c_{\infty} k .
$$

The coupling to the faster dynamic modes (connected with higher time derivatives of the longitudinal current) can only slightly renormalize down the theoretical dispersion law, however it will never result in the hydrodynamic speed of sound $c_{s}$ and positive sound dispersion [11]. Within the proposed in [1] theoretical approach is impossible to obtain the propagating modes with adiabatic speed of sound, because in order to obtain it one has to include coupling with density and energy (or heat) density fluctuations into the theoretical scheme. And, as it was expected from the wrong behavior of $C^{L}(k, \omega)$ discussed above, the proposed expression for dispersion of longitudinal collective excitations is wrong too. In Fig.4 only for two lowest $k$-values we obtained the positive expression under the square root in their Eq.(20). For higher wave numbers the expression under the square root became negative, i.e. no propagating modes for those wave numbers. It is not clear how in Fig.5 of [1] the authors were able to reproduce perfectly the MD data by using their Eq.20 and even reach the adiabatic speed of sound in the long-wavelength region, that is impossible to do in their theoretical approach. Even conceptually their theoretical approach, which does not contain coupling to fluctuations of conserved quantities, density $n(k, t)$ and energy density $e(k, t)$, and Eq.20 cannot result in the long-wavelength limit in the linear dispersion with the adiabatic speed of sound. In their run for the "overarching goal of this research programme" the authors forgot about the existing methodologies of calculations and theories of collective excitations in liquids, which correctly satisfy exact relations and a large number of sum rules.

Another point we want to discuss here is the claimed "gapped momentum states"[1]. It sounds strange that the authors are trying to represent the well known in the literature shear waves with a propagation gap as some special finding and rename them as the "gapped momentum states". The title of [1] stating "Collective modes and gapped momentum states ..." clearly discriminates between the "collective modes" and "gapped momentum states" that is not correct because there is no difference between ordinary collective shear waves and "gapped momentum states". Moreover, it has been known for long time that other collective propagating processes in liquids have very similar behavior of their dispersion, like heat waves $[10,12,17]$ or optic-like modes in binary liquids with demixing tendencies $[12,18]$. We would like to remind the readers that by 2017 the same group assured the 


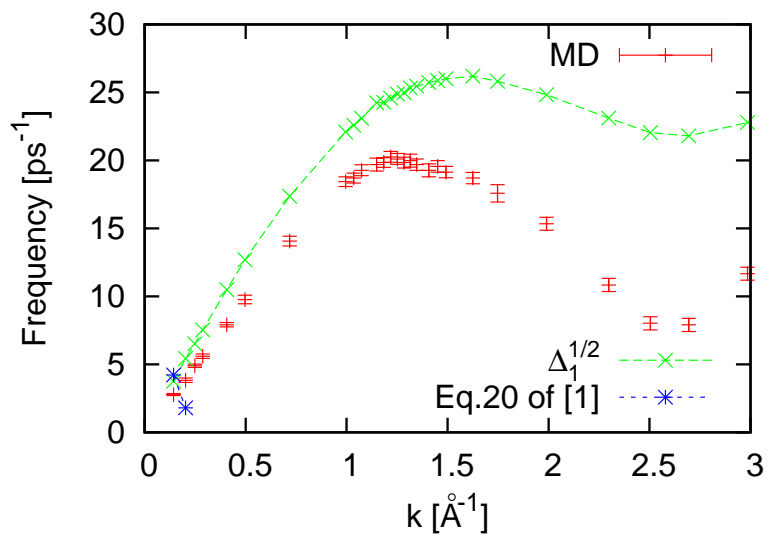

FIG. 4: Peak positions of the longitudinal current spectral function $C^{L}(k, \omega)$, obtained from MD simulation (plus symbols with error bars). The dispersion of the nondamped high-frequency acoustic-like modes with long-wavelength asymptote (2) is shown by line-connected cross symbols. Eq.20 of [1] (line-connected star symbols) contains positive expression unders square root only for two lowest $k$-points, for larger $k$-values no real $\omega_{c}^{L}(k)$ exist.

community in Frenkel-like dispersion of the transverse excitations in liquids[19, 20], i.e. when the transverse excitations in liquids exist only above the so-called Frenkel frequency cut-off, that contradicted the existed theories of transverse exsitations [4, 22, 23] and MD data (see our discussion in [16]), which evidenced on existing the long-wavelength propagation gap for shear waves. In 2017 the same authors revealed that the dispersion of shear waves indeed starts from zero frequency outside the propagation gap and published a paper [21] in which claimed that the propagation gap originates from the Frenkel jumps and is defined by the single-particle Frenkel time (quoting [21]: " $\tau$ is understood to be the full period of the particles jump motion equal to twice Frenkels $\left.\tau^{\prime \prime}\right)$. That claim again contradicted the existed theory of transverse excitations in liquids[4, 22, 23], in which the collective shear stress relaxation with Maxwell relaxation time is responsible for the propagation gap and we showed several times that there is huge difference between the collective and singleparticle relaxation processes in their effect on transverse dynamics[24, 25]. Now, in [1] the same group started to rename the ordinary shear waves of liquid dynamics as "gapped momentum states". 


\section{CONCLUSION}

The proposed in [1] theoretical scheme for description of longitudinal collective excitations in simple liquids is not consistent with hydrodynamics, because only one hydrodynamic variable, the longitudinal current, was used in that scheme, that rised questions whether the obtained in [1] expressions for longitudinal current spectral function $C^{L}(k, \omega)$ and for the dispersion of collective excitations are correct. We performed molecular dynamics simulatins on a simple supercritical Ne at $295 \mathrm{~K}$ and density $1600 \mathrm{~kg} / \mathrm{m}^{3}$ with a purpose of numerical check of these expressions.

We showed that the proposed in [1] expression for $C^{L}(k, \omega)$ does not have correct lowfrequency limit $C^{L}(k, \omega \rightarrow 0)$ and even diverges in the long-wavelength limit, that is wrong, while according to the continuity equation it must be $C^{L}(k, \omega=0) \equiv 0$. Why their Fig.4 shows perfect agreement of their theoretical $C^{L}(k, \omega)$ with MD data we cannot explain.

Within the proposed in [1] theoretical scheme it is impossible to recover the hydrodynamic dispersion law in $k \rightarrow 0$ limit and macroscopic adiabatic speed of sound, because the coupling of the longitudinal current with other fluctuations of conserved quantities is absent in that scheme. We checked the proposed in [1] expression for the dispersion of collective excitations and found that with increasing wave numbers the expression under square root in their Eq.20 becomes negative, i.e. wrong result. Why their Fig.5 shows perfect agreement between their theoretical expression and the MD-obtained dispersion of collective excitation, and even recovers the hydrodynamic linear dispersion law with $c_{s}$ we cannot explain. We would suggest the authors of [1] to show their similar checks for the correlators $\left\langle\ddot{J}_{L}(-k) J_{L}(k)\right\rangle$

and $\left\langle\dddot{J}_{L}(-k) \dot{J}_{L}(k)\right\rangle$ as we presented in Fig.1, as well as to reveal the $k$-dependence of their $\Delta_{i}(k)$. This defintely will allow to find out why the low-frequency limit of $C^{L}(k, \omega)$, their Eq.18, and the long-wavelength limit of $\omega_{c}^{L}(k)$, their Eq.20, do not correspond to the data in their Figs.4 and 5, respectively.

[1] R.M. Khusnutdinoff, C. Cockrell, O.A. Dicks, A.C.S. Jensen, M.D. Le, L. Wang, M.T. Dove, A.V. Mokshin, V.V. Brazhkin, K. Trachenko, Phys. Rev. B 101, 214312 (2020).

[2] J.R.D. Copley, S.W. Lovesey, Rep. Prog. Phys. 38, 461 (1975).

[3] T. Scopigno, G. Ruocco and F. Sette, Rev.Mod.Phys., 77, 881 (2005). 
[4] J.-P.Hansen and I.R.McDonald, Theory of Simple Liquids (London: Academic) (1986).

[5] T. Scopigno, U. Balucani, G. Ruocco and F. Sette, J. Phys.:Condens. Matter, 12, 8009 (2000).

[6] I.P. Omelyan, I.M. Mryglod, M.V. Tokarchuk, Phys.Rev.E 57, 6667 (1998)

[7] J.-P.Boon and S.Yip, Molecular Hydrodynamics (New-York: McGraw-Hill) (1980).

[8] I.M.deSchepper, E.G.D.Cohen, C.Bruin, J.C. van Rijs, W.Montfrooij, and L.A. de Graaf, Phys. Rev. A 38, 271 (1988).

[9] I.M.Mryglod, I.P.Omelyan, and M.V.Tokarchuk, Mol. Phys. 84, 235 (1995).

[10] T. Bryk and I. Mryglod, Phys. Rev. E 63, 051202 (2001).

[11] T. Bryk, I. Mryglod, T. Scopigno, G. Ruocco, F. Gorelli and M. Santoro, J.Chem.Phys. 133, $024502(2010)$.

[12] T. Bryk, Eur. Phys. J. Spec. Top. 196, 65 (2011); 227, 2689 (2019).

[13] T. Bryk, G. Ruocco, T. Scopigno, J. Chem. Phys. 138, 034502 (2012).

[14] T. Bryk and I. Mryglod, Phys. Rev. E 64, 032202 (2001).

[15] T. Bryk and I. Mryglod, J. Phys.: Condens. Matter. 13, 1343 (2001).

[16] T. Bryk, F.A. Gorelli, I. Mryglod, G. Ruocco, M. Santoro, T. Scopigno, J. Phys. Chem. Lett. 8, 4995 (2017).

[17] D.D. Joseph, L. Preziosi, Rev. Mod. Phys. 61, 41 (1989).

[18] T. Bryk and I. Mryglod, J. Phys.: Condens. Matter. 14, L445 (2002).

[19] V.V. Brazhkin, Y.D. Fomin, A.G. Lyapin, V.N. Ryzhov, K. Trachenko, Phys.Rev. E 85, $031203(2012)$.

[20] L. Wang, C. Yang, M.T. Dove, Y.D. Fomin, V.V. Brazhkin, K. Trachenko, Phys.Rev. E 95, 032116 (2017).

[21] C. Yang, M.T. Dove, V.V. Brazhkin, K. Trachenko, Phys.Rev. Lett. 118, 215502 (2017).

[22] R.A.MacPhail, D.Kivelson, J. Chem. Phys. 80, 2102 (1984).

[23] T. Bryk and I. Mryglod, J. Phys.: Condens. Matter. 12, 6063 (2000).

[24] T. Bryk, I. Mryglod, G. Ruocco, T. Scopigno, Phys.Rev. Lett. 120, 219601 (2018).

[25] T. Bryk, F.A. Gorelli, I. Mryglod, G. Ruocco, M. Santoro, T. Scopigno, J.Phys.Chem. B. 122, 6120 (2018). 